\title{
A Pointer Theory Explanation of Weak Value Persistence Occurring in the Quantum Three Box Experimental Data
}

\author{
A.D. PARKS* And S.E. Spence \\ Electromagnetic and Sensor Systems Department, Naval Surface Warfare Center Dahlgren Division, \\ Dahlgren, VA 22448, USA \\ (Received September 20, 2016)
}

\begin{abstract}
A concise exact pointer theory for von Neumann projector measurements of pre- and post-selected quantum systems has previously been used to formalize the notion of weak value persistence and apply it to explain pointer position data collected from a dynamical quantum non-locality detection experiment. This paper applies this exact pointer theory to provide an operational explanation of weak value persistence observed in the data obtained from a recent quantum box experiment.
\end{abstract}

DOI: 10.12693/APhysPolA.130.1265

PACS/topics: 03.65.-w, 03.65.Ta, 03.65.Ca

\section{Introduction}

The theoretical notion of the weak value $A_{w}$ of a quantum mechanical observable $A$ was introduced by Aharonov et al. [1-3] nearly three decades ago. This quantity is the statistical result of a standard measurement procedure performed upon a pre-selected and postselected (PPS) ensemble of quantum systems when the interaction between the measurement apparatus and each system is sufficiently weak. Unlike the standard strong measurement of a quantum mechanical observable which significantly disturbs the measured system (i.e., "collapses" the wave function) and yields the mean value $\langle A\rangle$ as the measured value of $A$, a weak measurement of an observable for a PPS system does not appreciably disturb the quantum system and yields $A_{w}$ as the measured value for $A$. While the interpretation of weak values remains somewhat controversial, several of the unusual properties predicted by weak value theory have been experimentally verified, e.g. [4-6].

Projection operators, i.e. projectors, are an important part of the mathematical formalism of quantum mechanics. The measurement and interpretation of the weak values of projectors have also played central roles in recent experimental observations of dynamical quantum nonlocality-induced effects, e.g. [7, 8], as well as in the theoretical and experimental resolution of "Hardy's paradox", e.g. $[9,10]$, and the "quantum box problem", e.g. [11, 12]. Because of the significance and experimental utility of projectors, a concise exact theory for projector measurement pointers for both PPS and pre-selected only (PSO) systems has recently been developed [13].

In 2004 Resch et al. successfully performed an impressive optical experiment using weak values of projectors that verified the counterintuitive theoretical predictions of the quantum three box gedanken problem [12].

\footnotetext{
${ }^{*}$ corresponding author; e-mail: allen.parks@navy.mil
}

As an aside, they noted that two of the three projectors measured during the experiment maintained their unit weak values well beyond the boundaries that defined their weak measurement regime (i.e., their weak values persisted) and correctly pointed to the discussion in [11] as the theoretical explanation for this persistence (i.e., if a strong measurement of $A$ is $a$ with unit probability, then the associated weak measurement of $A$ will also be $a$ ). The purpose of this paper is to provide an (also correct) alternative operational explanation (i.e., more akin to experimental actuality) for this persistence strictly in terms of the concise exact pointer theory for projector measurements elaborated in [13].

\section{Weak measurements and weak values}

Weak values arise in the context of the von Neumann description of a quantum measurement at time $t_{0}$ of a time-independent observable $A$ that describes a quantum system in an initial fixed pre-selected state $\left|\psi_{i}\right\rangle=\sum_{J} c_{j}\left|a_{j}\right\rangle$ at $t_{0}$, where the set $J$ indexes the eigenstates $\left|a_{j}\right\rangle$ of $\widehat{A}$ with $\widehat{A}\left|a_{j}\right\rangle=a_{j}\left|a_{j}\right\rangle$. In this description the Hamiltonian operator for the interaction between the measurement apparatus (i.e., the measurement pointer) and the quantum system is

$$
\widehat{H}=\gamma(t) \widehat{A} \widehat{p} .
$$

Here $\gamma(t)=\gamma \delta\left(t-t_{0}\right)$ defines the strength of the impulsive measurement interaction at $t_{0}$ and $\widehat{p}$ is the momentum operator for the pointer which is in the initial state $|\phi\rangle$. Let $\widehat{q}$ be the pointer's position operator that is conjugate to $\widehat{p}$ and assume that $\langle q \mid \phi\rangle \equiv \phi(q)$ is real valued.

Prior to the measurement the pre-selected system and the pointer are in the tensor product state $\left|\psi_{i}\right\rangle|\phi\rangle$. Immediately following the measurement the combined system is in the state

$$
|\Phi\rangle=\mathrm{e}^{-\frac{i}{\hbar} \int \widehat{H} \mathrm{~d} t}\left|\psi_{i}\right\rangle|\phi\rangle=\mathrm{e}^{-\frac{i}{\hbar} \gamma \widehat{A} \widehat{p}}\left|\psi_{i}\right\rangle|\phi\rangle,
$$

where $\mathrm{e}^{-\frac{i}{\hbar} \gamma \widehat{A} \widehat{p}}$ is the von Neumann interaction operator. If the state $\left|\psi_{f}\right\rangle,\left\langle\psi_{f} \mid \psi_{i}\right\rangle \neq 0$, is post-selected at $t_{0}$, the 
resulting pointer state is

$$
|\Psi\rangle=\left\langle\psi_{f} \mid \Phi\right\rangle=\left\langle\psi_{f}\left|\mathrm{e}^{-\frac{i}{\hbar} \gamma \widehat{A} \widehat{p}}\right| \psi_{i}\right\rangle|\phi\rangle .
$$

Of course the PPS states are selected at times $t_{i}<t_{0}<t_{f}$ and must be evolved forward and backward in time, respectively, to the measurement time $t_{0}$.

A weak measurement of $A$ occurs when the interaction strength $\gamma$ is sufficiently small so that the system is essentially undisturbed by the measurement and the pointer's position uncertainty $\Delta q$ is much larger than the separation between $\widehat{A}$ 's eigenvalues. In this case the last equation becomes

$$
\begin{aligned}
& |\Psi\rangle \approx\left\langle\psi_{f}\left|\widehat{1}-\frac{\mathrm{i}}{\hbar} \gamma \widehat{A} \widehat{p}\right| \psi_{i}\right\rangle|\phi\rangle \\
& \text { or }|\Psi\rangle \approx\left\langle\psi_{f} \mid \psi_{i}\right\rangle \widehat{T}\left(\gamma A_{w}\right)|\phi\rangle,
\end{aligned}
$$

where

$$
A_{w} \equiv\left\langle\psi_{f}|\widehat{A}| \psi_{i}\right\rangle /\left\langle\psi_{f} \mid \psi_{i}\right\rangle \equiv\left(A^{1}\right)_{w}
$$

is the complex valued weak value of observable $A$ and the operator

$$
\widehat{T}\left(\gamma A_{w}\right) \equiv \mathrm{e}^{-\frac{\mathrm{i}}{\hbar} \gamma A_{w} \widehat{p}}
$$

is the translation operator for $|\phi\rangle$ defined by the action

$$
\left\langle q\left|\widehat{T}\left(\gamma A_{w}\right)\right| \phi\right\rangle=\phi\left(q-\gamma \operatorname{Re} A_{w}\right)
$$

and yielding

$$
|\langle q \mid \Psi\rangle|^{2} \approx\left|\left\langle\psi_{f} \mid \psi_{i}\right\rangle\right|^{2}\left|\phi\left(q-\gamma \operatorname{Re} A_{w}\right)\right|^{2}
$$

as the associated pointer distribution profile. Thus, after the measurement the final mean pointer position is

$$
\langle\Psi|\widehat{q}| \Psi\rangle=\langle\phi|\widehat{q}| \phi\rangle+\gamma \operatorname{Re} A_{w},
$$

i.e., the pointer is translated from its initial mean position $\langle\phi|\widehat{q}| \phi\rangle$ by the amount $\gamma \operatorname{Re} A_{w}$. When $\left\langle\psi_{f} \mid \psi_{i}\right\rangle$ is small, then $\operatorname{Re} A_{w}$ can lie far outside the eigenspectral limits of $\widehat{A}$ and the associated pointer shift is large. It should be noted that under certain circumstances the imaginary part $\operatorname{Im} A_{w}$ can be used to control the variance of the pointer state [14].

In order for measurements to qualify as weak measurements, the associated momentum uncertainty of the pointer must simultaneously satisfy the following two formal weakness conditions which define the extreme boundary of the weak measurement regime, e.g. [8]:

$$
\Delta p \ll \frac{\hbar}{\gamma}\left|A_{w}\right|^{-1}
$$

and

$$
\Delta p \ll \min _{(n=2,3, \ldots)} \frac{\hbar}{\gamma}\left|\frac{A_{w}}{\left(A^{n}\right)_{w}}\right|^{1 /(n-1)} .
$$

When measurements are performed in accordance with these inequalities, i.e. when they are well within the extreme boundary of the weak measurement regime, they are weak measurements and are said to be performed in the weak measurement regime. Measurements performed outside the weak measurement regime are strong measurements and are not considered to be weak measurements.

\section{Exact pointer theory for projector measurements of PPS systems: an overview}

Let $\widehat{A}$ be a projection operator and observe that since $\widehat{A}^{n}=\widehat{A}, n \geq 1$, the von Neumann interaction operator is given exactly by

$$
\mathrm{e}^{-\frac{\mathrm{i}}{\hbar} \gamma \widehat{A} \widehat{p}}=\widehat{1}-\widehat{A}+\widehat{A} \widehat{S},
$$

where $\widehat{S} \equiv \mathrm{e}^{-\frac{\mathrm{i}}{\hbar} \gamma \widehat{p}}$ is defined by its action $\langle q|\widehat{S}| \phi\rangle \equiv$ $\phi(q-\gamma)$ upon the initial pointer state $|\phi\rangle$. Substitution of Eq. (4) into Eq. (1) and normalizing the result gives

$$
|\Theta\rangle=\frac{\mathrm{e}^{\mathrm{i} \chi}}{N}\left(1-A_{w}+A_{w} \widehat{S}\right)|\phi\rangle
$$

as the exact normalized pointer state that results from a measurement of $A$. Here

$$
\begin{aligned}
& \mathrm{e}^{\mathrm{i} \chi} \equiv \frac{\left\langle\psi_{f} \mid \psi_{i}\right\rangle}{\left|\left\langle\psi_{f} \mid \psi_{i}\right\rangle\right|}, \\
& N=\sqrt{a+J(\widehat{1})}, \\
& a=1-2 \operatorname{Re} A_{w}+2\left|A_{w}\right|^{2}
\end{aligned}
$$

and

$$
\begin{aligned}
& J(\widehat{x})=A_{w}\left(1-A_{w}^{*}\right)\langle\phi|\widehat{x} \widehat{S}| \phi\rangle+A_{w}^{*}\left(1-A_{w}\right)\langle\phi|\widehat{S} \widehat{x}| \phi\rangle, \\
& x=1, q .
\end{aligned}
$$

Using Eq. (5) the exact pointer position after the measurement is readily found to be

$$
\langle\Theta|\widehat{q}| \Theta\rangle=\left(\frac{1}{N^{2}}\right)\left[a\langle\phi|\widehat{q}| \phi\rangle+J(\widehat{q})+\gamma\left|A_{w}\right|^{2}\right] .
$$

Note the succinct form of Eq. (6) and - more importantly - the fact that no approximations have been used to obtain it. Thus, Eq. (6) is not only exact - it is also valid for any interaction strength $\gamma$.

Of particular interest here is the fact that when $A_{w}=1$ the exact mean pointer position given by Eq. (6) for any interaction strength is precisely equal to the mean pointer position given by Eq. (3) obtained from a weak value measurement of $A$ performed in the weak measurement regime (a similar argument also holds for $A_{w}=0$ ). To see this, observe that when $A_{w}=1$, then $a=1, J(\widehat{q})=0=$ $J(\widehat{1})$, so that $N=1$ and

$$
\langle\Theta|\widehat{q}| \Theta\rangle=\langle\phi|\widehat{q}| \phi\rangle+\gamma=\langle\Psi|\widehat{q}| \Psi\rangle,
$$

where the second equality also applies only in the weak measurement regime.

The exact mean pointer position is a linear function of interaction strength and has $\langle\phi|\widehat{q}| \phi\rangle$ as its " $y$-intercept" and $A_{w}=1$ as its "slope". Since $A_{w}=1$ regardless of the magnitude of $\gamma$, the pointer is said to exhibit weak value persistence, i.e. $A_{w}=1$ persists outside the weak measurement regime where the measurements are no longer weak measurements.

\section{The quantum three box experiment}

The quantum three box problem is an example of a PPS quantum system that exhibits counterintuitive 
behavior, e.g. [11]. In the Resch et al. experimental implementation of the quantum three box problem a particle is prepared at time $t_{i}$ in a pre-selected state $\left|\psi_{i}\right\rangle=\sqrt{2 / 5}|A\rangle+\sqrt{2 / 5}|B\rangle+\sqrt{1 / 5}|C\rangle$ that is the superposition of being in one of three orthogonal boxes $A, B$, and $C$ with probabilities $2 / 5,2 / 5$, and $1 / 5$, respectively, and is post-selected at time $t_{f}$ to be in the state $\left|\psi_{f}\right\rangle=(1 / 2)|A\rangle+(1 / 2)|B\rangle-(1 / \sqrt{2})|C\rangle$. Using these PPS states (in this case they remain fixed when evolved to the time of measurement $t_{0}$ ) in Eq. (2) the theoretically predicted weak values for the projectors $\widehat{P}_{A} \equiv|A\rangle\left\langle A\left|, \widehat{P}_{B} \equiv\right| B\right\rangle\langle B|$, and $\widehat{P}_{C} \equiv|C\rangle\langle C|$ - which correspond to the weak probabilities for finding the particle in the respective boxes at the intermediate time of weak measurement $t_{i}<t_{0}<t_{f}$ - are readily found to be $P_{A w}=1, P_{B w}=1$, and $P_{C w}=-1$. Clearly, $P_{C w}$ is counterintuitive since it is negative and lies outside the normal range of positive values for classical probabilities. Vaidman refers to the weak probabilities $P_{A w}=1, P_{B w}=1$, and the sum $P_{A w}+P_{B w}+P_{C w}=1$ as three elements of reality for the particle [11].

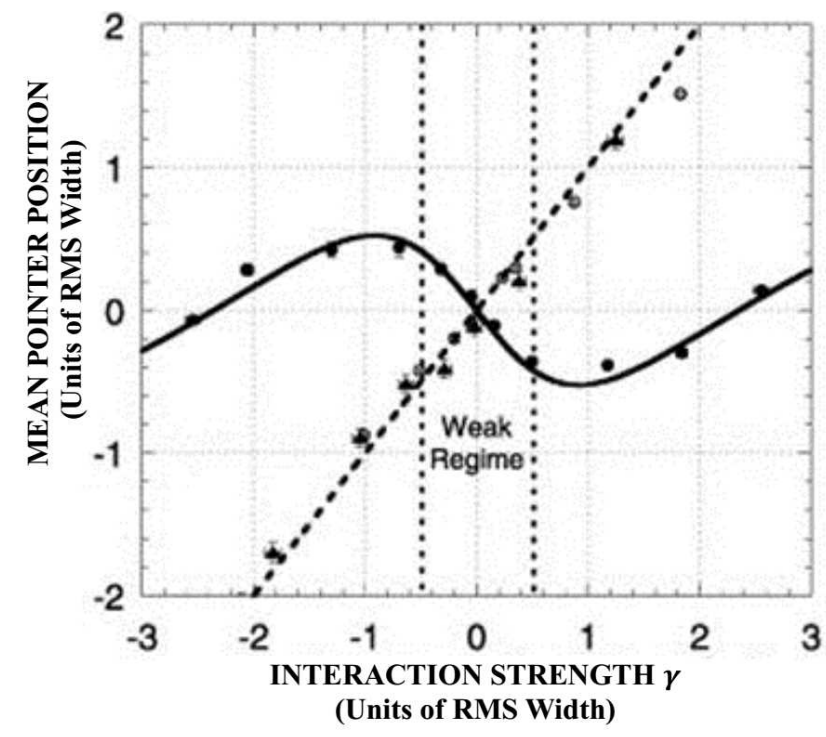

Fig. 1. Experimental data for weak value measurements of $\widehat{P}_{A} \widehat{P}_{B}$ and $\widehat{P}_{C}[12]$.

The Resch et al. experiment verified these theoretical predictions. Since the purpose of this paper is only to provide an explanation of the weak value persistence features identified in their data from the operational perspective of measurement pointer response, no discussion concerning their experimental apparatus or procedure is provided here (the interested reader is invited to consult [12] for details concerning the experiment). To this end consider the relevant (to this paper) part of their experimental data presented in Fig. 1. There mean pointer positions in rms beam width units obtained for weak measurements of $\widehat{P}_{A} \widehat{P}_{B}$ and $\widehat{P}_{C}$ (shown as open circles, solid triangles, and solid circles, respectively, in Fig. 1) are plotted for various measurement interaction strengths - also expressed in rms beam width units. The interaction strengths for which the measurements are weak - i.e., the weak measurement regime - are contained within the interval ranging from -0.5 to $+0.5 \mathrm{rms}$ beam width units and is delineated in Fig. 1 by vertical dashed lines.

A curve is fit to the weak value measurement pointer position data for $\widehat{P}_{C}$ and it is readily found that the slope of the linear portion of the curve contained within the weak measurement regime is -1 . As per Eq. (3), this slope corresponds to the weak value $P_{C w}$ and confirms the correctness of its theoretically predicted value. Also, as predicted by theory and as required by Eq. (3), the measured pointer positions for $P_{A w}$ and $P_{B w}$ both follow the dashed theoretical line of slope 1 within the weak measurement regime and have unit values. Thus, $P_{A w}=$ $1=P_{B w}$ and the theoretical predictions are completely confirmed.

Of interest here is the fact that these pointer positions continue to track this dashed line outside the weak measurement regime where the measurements become increasingly stronger as $|\gamma|>0.5$ and can no longer qualify as weak measurements - i.e., the weak values $P_{A w}=1=P_{B w}$ persist beyond the weak measurement regime. This behavior has been explained theoretically using the Aharonov, Bergmann, and Lebowitz formalism [15]. In particular, it has been proven theoretically that [16] "if the weak value of a dichotomic variable equals to one of its eigenvalues, then the outcome of a strong measurement of this variable is equal to that eigenvalue with probability one". This clearly applies here to both $\widehat{P}_{A}$ and $\widehat{P}_{B}$ since they are projectors and their unit weak value is one of their two eigenvalues (recall that 1 and 0 are the only two eigenvalues for any projector).

A direct and precise operational explanation based upon the exact properties of the experimental measurement pointer can also be provided for this persistence. Let us recall from Sect. 3 that Eq. (6) is the exact (i.e., no approximations are made) expression for the mean pointer position for a projector measurement of a PPS system that is applicable for any interaction strength. As was shown there, when the weak value of the projector being measured is 1 , the associated exact mean pointer position for any interaction strength is identical to the weak value theoretical mean pointer position for measurements in the weak measurement regime. This fact is expressed by Eq. (7) which clearly shows that the exact mean pointer position varies linearly with interaction strength. In particular, the line has unit slope (corresponding to $P_{A w}=1=P_{B w}$ in the experiment) with the initial mean pointer position (which is zero in the experiment) as its " $y$-intercept".

Thus, the persistence of the mean pointer positions for the $P_{A w}=1=P_{B w}$ measurements in the experiment is predicted exactly by Eq. (7). Since it exhibits a smooth linear behavior for pointer position within and outside the weak measurement regime, this prediction 
is completely consistent for projectors - from an operational pointer perspective - with the above statement in italics, i.e., Eq. (7) accurately reflects the projector's unit weak value within the weak measurement regime and its strongly measured unit value outside the weak measurement regime. Consequently, the following statement has been demonstrated to provide an alternative operational explanation of weak value persistence for the projectors measured in the quantum box experiment in terms of pointer position: "the mean pointer position associated with a projector measurement of a PPS system is given exactly by Eq. (7) for an arbitrary interaction strength when the projector's weak value is 1 ".

\section{Concluding remarks}

Equation (7) and the experimental data in Fig. 1 for the $P_{A w}=1=P_{B w}$ measurements both imply that these weak values will persist indefinitely with increasing interaction strength. Equation (7) also shows that there can be no persistence associated with the $\widehat{P}_{C}$ pointer position measurements. This is readily apparent in Fig. 1 where - outside the weak measurement regime - the data points converge to the mean value $\left\langle\psi_{i}\left|\widehat{P}_{C}\right| \psi_{i}\right\rangle=1 / 5$ as the interaction strength increases.

\section{Acknowledgments}

This research was supported by a grant from the Naval Surface Warfare Center Dahlgren Division's Inhouse Laboratory Independent Research Program.

\section{References}

[1] Y. Aharonov, D. Albert, A. Casher, L. Vaidman, New Techniques and Ideas in Quantum Measurement Theory, New York Academy of Science, New York 1986.

[2] Y. Aharonov, D. Albert, L. Vaidman, Phys. Rev. Lett. 60, 1351 (1988).

[3] Y. Aharonov, L. Vaidman, Phys. Rev. A 41, 11 (1990).

[4] N. Ritchie, J. Storey, R. Hulet, Phys. Rev. Lett. 66, 1107 (1991).

[5] A. Parks, D. Cullin, D. Stoudt, Proc. R. Soc. Lond. A 454, 2997 (1998).

[6] Q. Wang, F. Sun, Y. Zhang, L. Jian, Y. Huang, G. Guo, Phys. Rev. A 73, 023814 (2006).

[7] J. Tollaksen, Y. Aharonov, A. Casher, T. Kaufherr, S. Nussinov, New J. Phys. 12, 013023 (2010).

[8] S. Spence, A. Parks, Found. Phys. 42, 803 (2012).

[9] Y. Aharonov, A. Botero, S. Popescu, B. Reznik, J. Tollaksen, Phys. Lett. A 301, 130 (2002).

[10] J. Lundeen, A. Steinberg, Phys. Rev. Lett. 102 , 020404 (2009).

[11] L. Vaidman, Found. Phys. 26, 895 (1996).

[12] K. Resch, J. Lundeen, A. Steinberg, Phys. Lett. A 324, 125 (2004).

[13] A. Parks, S. Spence, J. Gray, Proc. R. Soc. Lond. A 470, 20130651 (2014).

[14] A. Parks, J. Gray, Phys. Rev. A 84012116 (2011).

[15] Y. Aharonov, P. Bergmann, J. Lebowitz, Phys. Rev. 134, B1410 (1964).

[16] Y. Aharonov, L. Vaidman, J. Phys. A Math. Gen. 24, 2315 (1991). 